\title{
EL CAMPO DE LA PRÁCTICA DOCENTE EN LA FORMACIÓN INICIAL. UN ESTUDIO DESCRIPTIVO DE UNA PROPUESTA CURRICULAR PARA LOS PROFESORADOS DE CIENCIAS EXACTAS \\ Y NATURALES
}

\author{
O CAMPO DA PRÁTICA DOCENTE NA FORMAÇÃO INICIAL. UM ESTUDO \\ DESCRITIVO DE UMA PROPOSTA CURRICULAR PARA OS PROFESSORADOS DE \\ CIÊNCIAS EXATAS E NATURAIS
}

THE FIELD OF TEACHING INTERNSHIP IN INITIAL TRAINING. A DESCRIPTIVE STUDY OF A CURRICULAR PROPOSAL FOR NATURAL SCIENCES TEACHER

TRAININGS

DOI: 10.22481/rbba.v1i02.7793

María Basilisa García

Universidad Nacional de Mar del Plata, Argentina ORCID: https://orcid.org/0000-0002-4282-6957

Endereço eletrônico: bachigarcia@gmail.com

Guillermo Cutrera

Universidad Nacional de Mar del Plata, Argentina ORCID: https://orcid.org/0000-0001-8452-4538

Endereço eletrônico: guillecutrera@gmail.com

\begin{abstract}
RESUMEN
En este trabajo presentamos y describimos los cambios proyectados en la estructura curricular para los profesorados de la FCEyN-UNMdP, en el contexto del proceso de acreditación en el que se inscribe la unidad académica. De los diferentes campos en los que se estructura la propuesta, nos detuvimos en el Campo de la Formación Práctica en el que, y con relación al plan de estudio vigente, se presentan los cambios de mayor significatividad. Presentamos, además, un dispositivo para el
\end{abstract}


seguimiento de las trayectorias formativas de los estudiantes en este Campo, considerando sus inserciones en las instituciones del nivel educativo. Ejemplificamos, finalmente, resultados de una investigación más amplia, centrada en el seguimiento de una futura profesora de Química.

Palabras clave: Formación docente inicial; Modelo interconectado de crecimiento profesional docente; Planes de estudio.

\title{
RESUMO
}

Neste trabalho apresentamos e descrevemos as modificações projetadas na estrutura curricular para os Professorados da FCEyN - UNMdP, (Faculdade de Ciências Exatas e Naturais da Universidade de Mar del Plata), no contexto do processo de acreditação no qual se inscreve a unidade acadêmica. Dos diferentes campos nos que se estrutura a proposta, detivemo-nos no Campo da Formação Prática no qual, e com a relação ao plano de estudo vigente, apresentam-se as mudanças de maior significância. Apresentamos, além disso, um dispositivo para o seguimento das trajetórias formativas dos estudantes neste Campo, considerando suas inserções nas Instituições do nível educativo. Exemplificamos, finalmente, resultados de uma pesquisa mais ampla, centrada no seguimento de uma futura Professora de Química.

Palavras-chave: Formação docente para a educação infantil; Modelo interconectado de crescimento profissional docente; Planos de estudo.

\begin{abstract}
In this extract we present and describe the projected changes in the curricular structure for the faculties FCEyN-UNMdP, in the context of the process of accreditation in which the academic unit is inscribed. Among the different areas in which the proposal is structured, we focused on the Field of Practical Formation where the changes of greater significance are presented in relation to the current syllabus. We also present a device for the follow-up of formative developments in this Field, taking into account their insert in institutions of a given educational level. Finally, we exemplify the results of a broader investigation centered in the follow-up of a future Chemistry teacher.
\end{abstract}

Keywords: Teacher training; Interconnected model of teacher professional growth; Study plans.

\section{INTRODUCCIÓN}

Las diferentes líneas de investigación que buscan atender las demandas de un nuevo modelo de formación docente para la enseñanza de las ciencias han aportado sólidos 
EL CAMPO DE LA PRÁCTICA DOCENTE EN LA FORMACIÓN INICIAL. UN ESTUDIO

DESCRIPTIVO DE UNA PROPUESTA CURRICULAR PARA LOS PROFESORADOS DE

CIENCIAS EXACTAS Y NATURALES

argumentos para repensarla en pos de formar profesionales que sean capaces de trasladar al aula una visión en línea con los intereses actuales de la disciplina y que sea capaz de transitar la distancia presente entre la escuela "existente", "real" y la pensada en las investigaciones. En este sentido, una de las críticas de mayor relevancia dirigida a los programas de formación docente inicial es su supuesta ineficiencia para construir instancias formativas que permitan a los futuros profesores disminuir la distancia entre la teoría y la práctica (YAYLI, 2008). Actualmente, el problema viene siendo recuperado desde líneas de investigación sobre la práctica docente que abordan su lectura a partir de diferentes maneras de pensar la relación teoría-práctica (HERRERA GONZÁLEZ Y MARTÍNEZ RUIZ, 2018). El desarrollo del conocimiento experto por parte de los docentes implica "teorizar el conocimiento práctico" y "poner en práctica el conocimiento teórico", haciendo explícito el conocimiento tácito que se obtiene de la experiencia y hacer interpretaciones personales del conocimiento. Estas interpretaciones, como plantea claramente MAINER BAQUÉ (2019), son resultado de una trayectoria escolar, que precede a la instancia de formación inicial y contribuye a construir un habitus profesional docente (PERRENOUD, 2004). Siguiendo a ALLIAUD (2007), los sujetos se constituyen social e históricamente, vinculados a sus historias personales. El tránsito por la escolaridad representa, por la continuidad y permanencia del sujeto en la institución escolar, una de las "fuentes" más relevantes en las actuaciones de los futuros profesores, manifestándose en patrones de pensamiento y acción, configurando su habitus profesional (SANJURJO, 2009), imprimiendo huellas frecuentemente implícitas, expresadas a modo de ideas, creencias, valoraciones sobre la realidad social, a menudo cristalizadas, a partir de los cuales reestructuran las cuestiones académicas que se abordan. Esto último exige pensar la formación desde una perspectiva superadora de modalidades tecnocráticas, diseñando programas de formación que promuevan la participación de los docentes en procesos reflexivos mediadores de sus prácticas de enseñanza (CHENG, TANG Y CHENG, 2012).

Partiendo de la centralidad que la relación teoría-práctica posee para el desarrollo profesional docente, y que los resultados de las investigaciones en el campo de la formación docente son un insumo indispensable para la el planteo de nuevos diseños curriculares, la Facultad de Ciencias Exactas y Naturales-UNMdP ha delineado una nueva propuesta curricular para las carreras de profesorados que ofrece. Esta propuesta plantea guiar y construir la trayectoria del futuro profesional docente y la instancia de socialización profesional a partir del seguimiento longitudinal de los procesos reflexivos que realiza.

Revista RBBA $\mid$ Revista Binacional Brasil Argentina 
EL CAMPO DE LA PRÁCTICA DOCENTE EN LA FORMACIÓN INICIAL. UN ESTUDIO

DESCRIPTIVO DE UNA PROPUESTA CURRICULAR PARA LOS PROFESORADOS DE

CIENCIAS EXACTAS Y NATURALES

Para poder llevar a la práctica esta nueva perspectiva de formación docente, se elaboraron proyectos de nuevos planes de estudio para las carreras de los profesorados en los que se incorporaron modificaciones sustanciales a los diseños vigentes. Los cambios fundamentales están centrados en una nueva propuesta de formación centrada en el Campo de la práctica, de carácter longitudinal e involucrando a asignaturas de la Formación General, de la Formación Pedagógica y de la Práctica Profesional Docente, articulando en ellas la teoría y la práctica.

En función de lo expuesto, en este trabajo se propone:

$\checkmark$ Presentar la estructura común del Campo de la Práctica elaborada para los nuevos planes de estudio de los profesorados en la FCEyN.

$\checkmark$ Describir las diferentes modalidades de articulación teoría-practica que se promueven en el contexto de la propuesta curricular de formación práctica.

$\checkmark$ Presentar un estudio de caso a modo de ejemplo de implementación de la nueva propuesta de formación.

Principales características de los nuevos proyectos de Planes de Estudios de las carreras de Profesorados de la UNMdP

Los nuevos planes de estudio de las carreras de profesorado están organizados alrededor de los siguientes campos de conocimiento: Formación Disciplinar Específica, Formación General, Formación Pedagógica y Formación Práctica. Este último campo es el que presenta los cambios más sustanciales ya que en él tienen lugar las Prácticas Profesionales Docentes (en adelante PPD) que se centran en el desarrollo de las capacidades para la actuación docente a través de la participación e integración progresiva de los estudiantes en los distintos contextos socioeducativos. En la Figura 1 se presentan la estructura del plan de estudio vigente y la correspondiente a la nueva propuesta curricular, cuyos Campos se caracterizan en los apartados siguientes. 
EL CAMPO DE LA PRÁCTICA DOCENTE EN LA FORMACIÓN INICIAL. UN ESTUDIO

DESCRIPTIVO DE UNA PROPUESTA CURRICULAR PARA LOS PROFESORADOS DE

CIENCIAS EXACTAS Y NATURALES
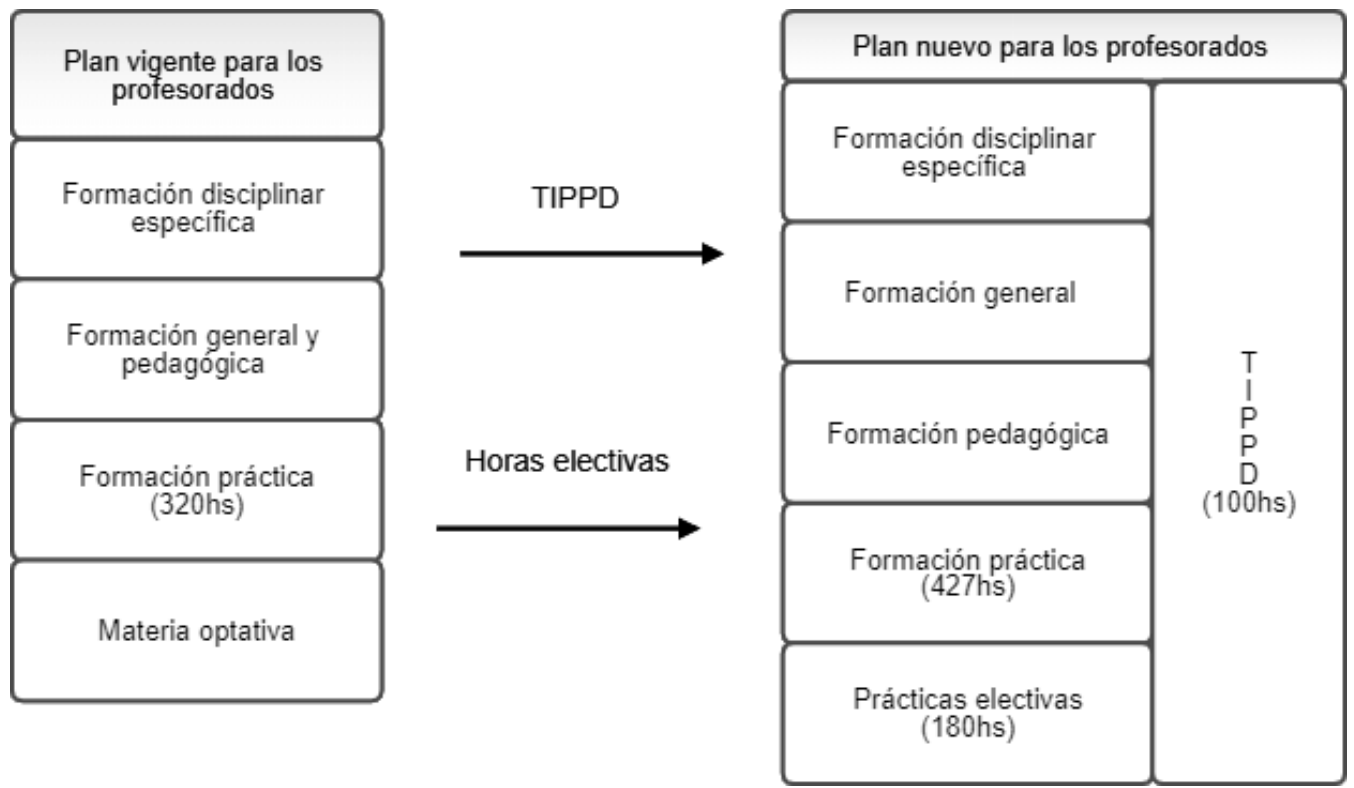

Figura 1: Cuadro comparativo de los planes de estudio vigentes con los nuevos

\section{El campo de la formación práctica}

Las PPD son prácticas sociales que se fundamentan en concepciones y valoraciones que nutren la acción y en las que teoría y práctica son mutuamente constitutivas e interactúan entre sí. Las prácticas docentes son abordadas y concebidas en su complejidad considerando sus diversas dimensiones: las relativas a cada campo específico de conocimiento que es objeto de enseñanza y las dimensiones sociales, históricas, políticas, culturales, filosóficas, epistemológicas, subjetivas, pedagógicas, didácticas y metodológicas. Por esta razón desarrollan un recorrido amplio en el plan de estudios, articulado en sucesivas etapas que culminan con la Residencia o Práctica Profesional. Se concretan principalmente mediante actividades que constituyen experiencias prácticas en distintos institucionales sociales, incluyendo los niveles educativos secundario y terciarios - universitario y no universitário del sistema educativo argentino.

Los propósitos principales de este campo de formación tienden a:

Desarrollar la comprensión del ejercicio de la profesión docente como una práctica social enmarcada en contextos sociales y culturales diversos.

Establecer un posicionamiento reflexivo y crítico respecto de los procesos involucrados en las propias prácticas, las razones y sentidos que las orientan y los efectos que producen, a partir de un conocimiento situado a nivel nacional y regional. 
Propiciar la valoración de la actividad profesional docente como una actividad social y colaborativa, orientada a aprender a pensar y a hacer con otros.

Lograr una formación sólida y de calidad integrando los conocimientos del campo del conocimiento disciplinar y del campo pedagógico, desde una posición de reflexión crítica.

Posibilitar la planificación, puesta en práctica y evaluación de propuestas de enseñanza y de aprendizaje pertinentes.

Favorecer la producción y la selección crítica de materiales didácticos.

A partir de la propuesta e implementación del Campo de la Formación Práctica se renuncia a considerar a la universidad como contexto formativo excluyente, avanzando en el reconocimiento que otras instituciones y otros profesionales poseen en la formación de la de los futuros profesionales docentes.

Debido a que la etapa de formación inicial de grado universitario tiene especial relevancia por su incidencia en la configuración de la identidad docente, debe poner en juego diversos tipos de saberes y conocimientos, asegurar su complementariedad e incluir distintos formatos y dispositivos didácticos. En base a esto, la nueva propuesta de plan de estudio plantea un aumento en la carga horaria de las PPD articulándolas con los demás campos de formación, diversificando la experiencia práctica, extendiéndola a distintos niveles de enseñanza (incluyendo el universitario) e integrándola al plan de estudios a partir de los primeros años.

En base a lo anterior, se propone organizar el campo de la práctica en tres modalidades:

$\checkmark$ Trayecto de las Prácticas Optativas y Electivas (POyE)

$\checkmark$ Trayecto de la Práctica Profesional Docente (PPD)

$\checkmark$ Trayectos de Iniciación a la Práctica Profesional Docente (TIPPD)

\section{Descripción de los trayectos Trayecto de las Prácticas Optativas y Electivas (POyE)}

Las Prácticas Electivas constituyen espacios formativos donde el estudiante, en función de sus intereses, puede optar por desarrollar tareas de aprendizaje que tienen un carácter menos estructurado como, por ejemplo, asistir a un congreso, participar en una pasantía de investigación y/o de extensión, o realizar una práctica socio comunitaria. Esto le da una característica más dinámica al plan de estudio y la posibilidad de una formación más amplia y diversa al futuro profesional docente.

Se entiende por prácticas optativas aquellas que se eligen dentro de un conjunto finito de alternativas ofrecidas en el currículo y por prácticas electivas aquellas que el estudiante 
EL CAMPO DE LA PRÁCTICA DOCENTE EN LA FORMACIÓN INICIAL. UN ESTUDIO

DESCRIPTIVO DE UNA PROPUESTA CURRICULAR PARA LOS PROFESORADOS DE

CIENCIAS EXACTAS Y NATURALES

puede seleccionar y proponer más allá de los contenidos específicos de su plan de estudios, siempre que sean previamente aprobadas por los responsables de este campo.

Ambas modalidades de prácticas, pertenecientes a este trayecto, podrán acreditarse a lo largo de la carrera, pero deberán ser completadas antes de la residencia docente.

\section{Trayecto de la Práctica Profesional Docente (PPD)}

Es el espacio de la práctica profesional docente propiamente dicha. Incluye dos asignaturas que en su conjunto, conforman la residencia: Prácticas Docentes I y Prácticas Docentes II.

La idea central de estos dos espacios pertenecientes a la práctica profesional es llevar a cabo diferentes intervenciones didácticas a partir de las cuales se generen contextos formativos que permitan la reflexión sobre la propia acción de manera tal que los futuros docentes vayan construyendo su conocimiento profesional sobre la base de la explicitación de sus propias concepciones, poniéndolas en diálogo permanente con los nuevos conocimientos que le va proporcionando la carrera y con la experiencia práctica. En este sentido, las prácticas de enseñanza se asumen como objeto de investigación, reinscribiendo el papel de las experiencias de la práctica en la formación profesional docente en una perspectiva interpretativa-crítica (COCHRAN-SMITH AND LYTLE, 1999). La formación inicial docente, y en especial en el contexto de la práctica, debe promover estrategias orientadas a proporcionar al futuro profesor oportunidades para poner en acción la reflexión metacognitiva, de manera que desencadene procesos de autorregulación en sus percepciones y acciones docentes. La "práctica" se constituye, entonces, en un espacio privilegiado de desarrollo del futuro profesor que reconoce su capacidad reflexiva y elaboradora de conocimiento profesional, así como la comprensión y el manejo de los contextos que condicionan su acción dentro y fuera de los centros escolares.

\section{Trayectos de Iniciación a la Práctica Profesional Docente (TIPPD)}

En la propuesta del plan de estudio se proponen cinco Trayectos para la inserción institucional, desarrollados desde los primeros años de la carrera de los profesorados en distintos niveles del sistema educativo. Los Trayectos son espacios curriculares involucran a los estudiantes en tareas que transitan desde los primeros acercamientos a las instituciones y a

Revista RBBA $\mid$ Revista Binacional Brasil Argentina 
EL CAMPO DE LA PRÁCTICA DOCENTE EN LA FORMACIÓN INICIAL. UN ESTUDIO DESCRIPTIVO DE UNA PROPUESTA CURRICULAR PARA LOS PROFESORADOS DE CIENCIAS EXACTAS Y NATURALES

los principales actores de la educación a intervenciones acotadas en el tiempo, con diferentes propósitos según la asignatura en la que está planteado el Trayecto. Cada uno de estos Trayectos consiste en un taller de 15 horas y se encuentra asociado con una asignatura específica del plan de estudios (Tabla.1).

\begin{tabular}{|l|l|}
\hline TIPPD & Asignatura \\
\hline TIPPD 1 & Teoría de la Educación \\
\hline TIPPD 2 & Psicología Educativa \\
\hline TIPPD 3 & Política, Organización y Gestión Educativa. \\
\hline TIPPD 4 & Didáctica General \\
\hline TIPPD 5 & Didáctica Específica \\
\hline
\end{tabular}

Tabla 1: Distribución de los TIPPD en las diferentes asignaturas.

Estos Trayectos complementan una perspectiva del Practicum como un recurso curricular cuya principal aportación reside en generar un contexto que permita una mayor integración entre teoría y práctica, entre aprendizajes disciplinares y aplicación del conocimiento en contextos profesionales reales. Desde esta perspectiva se pretende desarrollar su función formativa pensándolo en relación y formando parte de las diferentes materias que forman parte tanto del Campo de la Formación General como del Campo de la Formación Pedagógica.

Más allá de las particularidades de cada caso, los Trayectos tienen el propósito común de organizar y problematizar a partir de inserciones institucionales, los contenidos trabajados en la asignatura con la cuales están asociados, promoviendo prácticas reflexivas como herramienta analítica general. Con la incorporación de estos Trayectos se busca promover la articulación entre los contextos de teoría y práctica y llevar a cabo un seguimiento longitudinal de cada estudiante que permita recuperar estas articulaciones en un contexto más amplio.

En una primera aproximación, algunos de los propósitos asociados a los TIPPD serían los siguientes:

El TIPPD1: indagar y analizar aspectos socio-culturales y pedagógicos de la institución educativa y formará parte de la asignatura Teoría de la Educación. 
EL CAMPO DE LA PRÁCTICA DOCENTE EN LA FORMACIÓN INICIAL. UN ESTUDIO

DESCRIPTIVO DE UNA PROPUESTA CURRICULAR PARA LOS PROFESORADOS DE

CIENCIAS EXACTAS Y NATURALES

El TIPPD 2: analizar procesos de aprendizaje en las instituciones escolares y de las características de los sujetos que serán los futuros alumnos de los profesores en formación. Es un Trayecto integrado en la asignatura Psicología Educativa.

El TIPPD 3: estudiar desde un abordaje situado las cuestiones vinculadas con la escuela como institución. Es un Trayecto integrado en la asignatura Política, Organización y Gestión Educativa

El TIPPD 4: analizar y discutir cuestiones vinculadas con el rol docente y la enseñanza. Es un Trayecto integrado en la asignatura Didáctica General.

EL TIPPD 5: analizar y discutir cuestiones vinculadas con los diferentes dominios que componen el Conocimiento Didáctico del Contenido (Wilson, Borowski, \& van Driel, 2019) Es un Trayecto integrado en la asignatura Didáctica Específica.

\section{La integración curricular en el Campo de la Práctica}

La escalera de HARDEN (2000) marca los once escalones que transitan entre una estructura curricular constituida por unidades aisladas (isolation) y otra centrada en un planteamiento transdisciplinar o modular. En esta metáfora, los primeros cinco escalones (con variantes entre ellos) representan estructuras curriculares centradas en las materias basadas en asignaturas; los siguientes, intermedios, estructuras basados en asignaturas con actividades integradas y los últimos escalones, estructuras curriculares con asignaturas integradas.

Las alternativas que permitieron transitar hacia relaciones entre asignaturas que, recurriendo a la metáfora propuesta por HARDEN (2000), pueden ser caracterizadas por las modalidades denominadas de “conocimiento" y, progresivamente, “armonización”. En la primera de estas nuevas modalidades, -conocimiento o concienciación - los profesores de las diferentes materias accedieron a reconocer lo enseñado en las otras materias. Este conocimiento ofreció la posibilidad de evitar reiteraciones temáticas innecesarias en las diferentes materias de la formación especializada. No obstante, este conocimiento no necesariamente se traducía en avanzar hacia la posibilidad de recuperar temáticas convergentes, reconociendo la posibilidad de abordarlas desde perspectivas alternativas. El aislamiento perduraba en evitar un conocimiento con mayor profundidad no sólo en términos de lo trabajado sino, además, del abordaje metodológico utilizado. Sobre estas dificultades avanzó hacia la modalidad indicada armonización-, en el proceso "en acto" de las relaciones entre asignaturas en el Departamento \begin{tabular}{l|l} 
Revista RBBA & Revista Binacional Brasil Argentina
\end{tabular} 
EL CAMPO DE LA PRÁCTICA DOCENTE EN LA FORMACIÓN INICIAL. UN ESTUDIO

DESCRIPTIVO DE UNA PROPUESTA CURRICULAR PARA LOS PROFESORADOS DE

CIENCIAS EXACTAS Y NATURALES

de Educación Científica. Esta etapa de integración también ha sido denominada "conexión" (FOGARTY, 1991). Las disciplinas sostienen su identidad curricular; sin embargo, y a diferencia de la modalidad anterior, los profesores desarrollaron conexiones explícitas dentro del área temática con otras áreas temáticas y, además, cada profesor consultó con sus colegas a la hora de ir perfilando la propuesta curricular ofrecida a los estudiantes.

\section{Propuesta para el seguimiento de las trayectorias de los estudiantes}

En el presente trabajo, nos limitamos a ilustrar el seguimiento de estudiantes en los TIPPD y en la PPD. En línea con lo expuesto, se trabaja con un marco centrado en la reflexión como herramienta analítica general. Los procesos reflexivos de los futuros profesores, en cada TIPPD, son identificados y analizados en el marco del Modelo Interconectado de Crecimiento Profesional Docente (MICPD) diseñado por CLARKE Y HOOLINGSWORTH, (2002). El modelo describe los procesos de formación docente mediante cuatro dominios que interactúan entre sí por medio de procesos reflexivos. Estos son: el Dominio Personal (DPe), conformado por los conocimientos, creencias y actitudes de los docentes; el Dominio Externo (DE) conformado por fuentes externas de información y estímulos; el Dominio de la Práctica (DPr), que involucra la experimentación profesional en el aula y el Dominio de la Consecuencia (DC), conformado por resultados destacados relacionados con la práctica en el aula. A partir del MICPD se puede describir y analizar la construcción del conocimiento profesional de los futuros docentes en términos de cambios en los dominios descritos a través de dos mecanismos específicos: la promulgación y la reflexión. La Figura 2 esquematiza los dominios y las vías de conexión correspondientes a las relaciones entre ellos. 


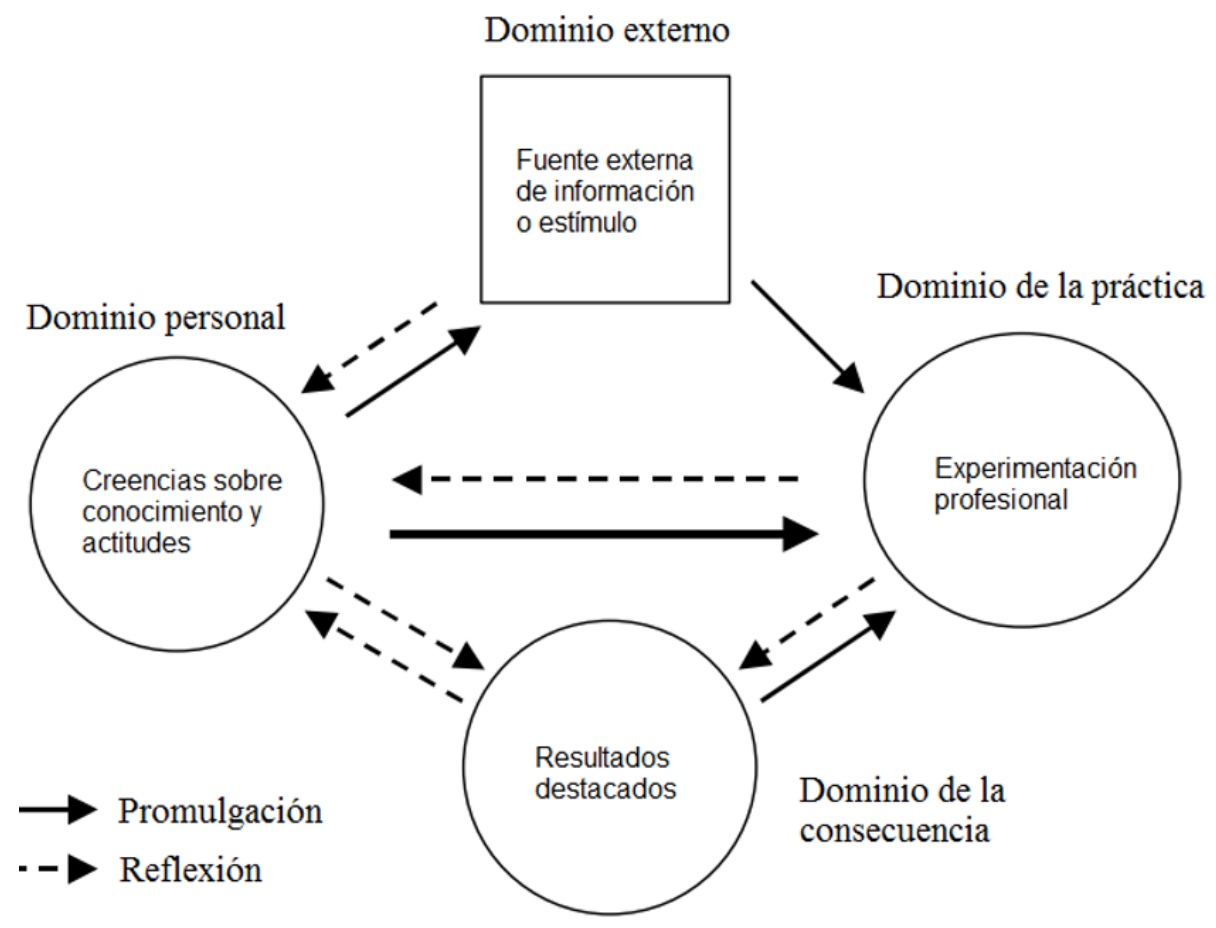

Figura 2: Dominios propuestos por el modelo interconectado de desarrollo profesional docente y relaciones entre ellos (Traducido y adaptado de CLARKE Y HOLLINSWORTH, 2002).

Para analizar cómo se desarrollan estos procesos en los estudiantes, entendemos a la acción reflexiva como una forma de afrontar y responder a los problemas a través de una consideración activa, persistente y cuidadosa de toda creencia o práctica realizada a la luz de los fundamentos que la sostienen y las consecuencias a las que conduce (DEWEY, 1989). La promulgación refiere a aquello que el estudiante hace o dice como resultado de lo que sabe, cree o ha experimentado; es una conducta no mediada por una reflexión (CLARKE Y HOLLINSWORTH, 2002). Los criterios que se establecieron para considerar cuándo se evidencia una relación, promulgación o reflexión, entre los dominios se tomaron de la propuesta de Justi y Van Driel (2006).

Cada uno de los dominios propuestos en el modelo se expresa con importancias relativas diferentes según las asignaturas relacionadas por los TIPPD. Las interacciones entre dominios se modifican durante el recorrido que el estudiante realiza al transitar de los TIPPD al campo de las PPD. Estos cambios se inscriben tanto en términos de los dominios, relativamente privilegiados en cada materia, como de las frecuencias en las relaciones que pueden establecerse entre dominios. Los cambios en el dominio personal de los estudiantes, durante los TIPPD, se 
EL CAMPO DE LA PRÁCTICA DOCENTE EN LA FORMACIÓN INICIAL. UN ESTUDIO

DESCRIPTIVO DE UNA PROPUESTA CURRICULAR PARA LOS PROFESORADOS DE

CIENCIAS EXACTAS Y NATURALES

relacionarían, en principio, con una mayor frecuencia dada por la presencia del Dominio Externo respecto del Dominio de la Práctica; estas relaciones se invertirían, en el campo de las PPD.

Los TIPPDs se constituyen en un ámbito formativo a partir del cual promover instancias reflexivas con los Dominios Personal, de la Práctica y de las Consecuencias. La naturaleza de estas relaciones puede inscribirse en la distinción entre las modalidades de reflexión en la acción y sobre la acción, respectivamente (SCHÖN, 1998). Además, las interacciones del estudiante con los profesores de las asignaturas asociadas a los TIPPDs y con sus pares delimitan instancias desde las cuales pensar y actuar en el Dominio de la Práctica. Estas instancias pueden ser construidas desde el trabajo sobre los marcos teóricos referenciales abordados en las asignaturas y desde la recuperación de las consecuencias relevantes derivadas del Dominio de la Práctica que, mediadas por instancias socializadoras con pares y profesores de la asignatura, pueden definir nuevas direcciones en las intervenciones institucionales.

En la última etapa del trayecto formativo, el campo de las prácticas profesionales docentes (PPD) presenta, con relación a los TIPPDs una instancia centrada en el Dominio de la Práctica. La transición de los TIPPD a las PPD en la estructura curricular se traduce en una mayor frecuencia de oportunidades para vincular el Dominio de la Práctica con los restantes Dominios a partir de la centralidad que las PPD. Las interacciones del Dominio Personal de los estudiantes se inscriben en relación al Dominio de la Práctica sea directamente o a través de las relaciones de este último con el de las Consecuencias relevantes. El Dominio Externo se estructura no solo a partir de los marcos teóricos trabajados que explicitan interpretaciones de la práctica docente sino, y especialmente, a partir de las instancias socializadoras entre pares. Esta última modalidad de relación del Dominio Externo con el Personal y de la Práctica se establece con mayor intensidad en el desarrollo de la residencia (Prácticas Docentes II).

\section{Seguimiento longitudinal de la formación de un estudiante a lo largo de del Campo de la Formación Práctica. Avance de resultados}

A modo de ejemplo, se presenta un estudio de caso que muestra el trabajo longitudinal realizado en los TIPPD con una estudiante de profesorado en química. Utilizando el MICPD, se analizó el modo en que la estudiante fue construyendo el conocimiento profesional en su recorrido por los diferentes TIPPD.

\begin{tabular}{l|l} 
Revista RBBA & Revista Binacional Brasil Argentina
\end{tabular} 
EL CAMPO DE LA PRÁCTICA DOCENTE EN LA FORMACIÓN INICIAL. UN ESTUDIO

DESCRIPTIVO DE UNA PROPUESTA CURRICULAR PARA LOS PROFESORADOS DE

CIENCIAS EXACTAS Y NATURALES

Se llevó a cabo un estudio de casos para describir y comprender las representaciones sobre la enseñanza en la estudiante del profesorado de Química, seguida de manera longitudinal en su recorrido por los TIPPD. Se escogió esta metodología porque permitió reflejar la peculiaridad de cada situación reflexiva a través de una descripción densa y profunda de la misma.

Para el análisis, el recorrido formativo se dividió en dos fases. La Fase 1 corresponde a las los TTPD 1, 2 y 3 (Tabla 1) asociados a asignaturas vinculadas con la formación general. La Fase 2 incluye los TTPD 4 y 5 (Tabla 1) y de las Prácticas Docentes e involucran las materias Didáctica General y Específica, Prácticas Docentes I y Prácticas Docentes II. En este apartado presentamos un adelanto de los resultados del seguimiento de una futura profesora de Química (que identificamos con el nombre "Ana") durante su trayecto formativo a través de estas dos Fases. Recuperamos las afirmaciones de la estudiante obtenidas en respuestas a actividades de foros (Fase 1) y a cuestionarios ReCo (LOUGHRAN, MULHALL Y BERRY, 2012) y entrevistas personalizadas (Fase 2).

Ana proviene de una familia de docentes. Sus concepciones sobre la educación, en general, sobre la enseñanza, sobre la formación docente, son atravesadas, entre otros, por su impronta familiar. Como señala HONG (2010), en su investigación centrada en docentes principiantes, algunas de ellas se expresan en prejuicios; en el caso de Ana, se manifiestan en su cuestionamiento a la utilidad de la formación pedagógica resignificada durante uno de los trayectos ("Al comienzo de la asignatura me encontraba un poco escéptica. Esta fue mi primera materia pedagógica y al venir de una familia de docentes el consenso en casa era que este tipo de materias "no servian para nada y eran aburridisimas", TIPPD 1). La resignificación de la formación pedagógica expresada por Ana permite ejemplificar la relación entre los dominios personal y externo, en términos de la modificación del primero por su participación en el dominios externos proporcionado por la materia.

Las afirmaciones de Ana, durante su tránsito por las asignaturas de los Campos de la Formación General y Pedagógica, expresaron, en algunas oportunidades, una mirada centrada en la dimensión política de la educación:

("[...] considero que el mayor desafío que tenemos como educadores de ciencia es bajar la ciencia al barrio. Muchas veces se considera que la ciencia es de unos pocos (de los inteligentes, los académicos, los que van a la universidad, las élites, las clases pudientes) cuando en realidad es de todos", TIPPD 1).

Revista RBBA $\mid$ Revista Binacional Brasil Argentina 
Estas ideas sobre la educación se desarrollaron de manera diferente cuando, durante esta misma Fase 1, justifica la importancia de enseñar un tema de Química ( "Es importante que un estudiante de secundario aprenda sobre uniones químicas porque es la base para luego comprender estructuras de Lewis que, a su vez, se vinculan con geometría electrónica y molecular y esto sirve para conocer las propiedades de los compuestos", TIPPD 2). La referencia a una temática disciplinar a ser enseñada contextualiza su respuesta, orientando su respuesta a la lógica disciplinar.

Esta tensión entre sus respuestas, según el contexto en el que éstas son ofrecidas, también se expresa en registros obtenidos en otros espacios del Campo Formativo de la Práctica. Su trabajo en la asignatura Didáctica de la Química implicó, entre otras actividades, la respuesta al instrumento ReCo sobre "Cinética Química". Las preguntas ofrecidas en el instrumento posiblemente orientaron sus respuestas a una lectura centrada en el qué y cómo enseñar, entre otros aspectos, diferenciando la mirada inicial sobre el proceso educativo. Si bien en sus respuestas Ana parece expresar una idea sobre la enseñanza y sobre el aprendizaje de la química priorizando la formación de ciudadanos, frente a la pregunta más concreta respecto al quehacer áulico, recupera una perspectiva en la que prioriza la estructura disciplinar (BAENA, 2000). Ana es consciente de la influencia formativa que, en ella, presenta su tránsito por la formación académica, al responder al ítem “¿Cuáles otros factores crees que influyen en la enseñanza del tema?” ("[...] no tengo ningún recuerdo de cómo veía el tema en la secundaria y dispongo de un entendimiento muy tradicional y teórico con el que debo romper para poder acercarme a los estudiantes”).

Esta aparente contradicción entre el decir y el proponer, expresada en sus respuestas, también aparece ante la pregunta de la ReCo ¿Qué conocimiento acerca del pensamiento de los estudiantes influye en tu enseñanza del tema? plantea la importancia de vincular los contenidos con el mundo real y lo provechoso de "encontrar" la química en él, evidenciando una visión diferente ( "En particular me interesa el vínculo con el mundo real [...]”). No obstante, al referir a la importancia que, para los estudiantes, tiene el aprendizaje de los conceptos, centra su respuesta en la lógica disciplinar ("Porque es la base de la mayoría de los contenidos que verán más adelante a medida que avancen en su escolaridad y ayuda a comprender la idea de cambio químico"). 
EL CAMPO DE LA PRÁCTICA DOCENTE EN LA FORMACIÓN INICIAL. UN ESTUDIO

DESCRIPTIVO DE UNA PROPUESTA CURRICULAR PARA LOS PROFESORADOS DE

CIENCIAS EXACTAS Y NATURALES

Más tarde, durante la asignatura "Prácticas Docentes I de Química" el trabajo realizado por Ana permitió observar que del predominio inicial de una visión propedéutica sobre la enseñanza de la química, centrada en la relevancia de lo disciplinar, transita hacia una perspectiva en la que, coexistiendo con esta representación (“Algunos conceptos como el balanceo de ecuaciones son necesarios para la construcción del conocimiento con el fin de avanzar a niveles superiores”), recupera la centralidad a la enseñanza de la disciplina como una herramienta para la formación de ciudadanos (“[...] les da un mejor entendimiento del mundo que los rodea y los fomenta a pensarlo con mayor curiosidad") incluyendo, además, una nueva lectura sobre la importancia de la enseñanza de la temática que relaciona ambas perspectivas ("A su vez el acercamiento a la ciencia como modelos fomenta una visión científica más moderna y no absolutista”). Estos resultados evidencian una re-descripción representacional respecto de la concepción dependiente de la enseñanza (BAENA, 2000) reflejada en un principio y, además, cierta convergencia entre el decir y el hacer en la etapa final de la asignatura. Por otra parte, expresan cambios en su Dominio Personal tanto a partir de su relación con el Dominio de la Práctica (a partir de sus intervenciones en el aula) como en su vínculo al Dominio Externo, proporcionado por el trabajo con docentes y pares en la asignatura. ${ }^{i}$ En una entrevista posterior, es posible recuperar esta redescripción en dos aspectos principales. Por un lado, en la relevancia que el contenido posee para la continuidad en prácticas de enseñanza según la prescripción curricular (“Tienen que aprender a balancear para poder seguir avanzando en el resto de los contenidos de la educación secundaria"); por otra parte, en la importancia que presenta su contextualización, pensando en la formación ciudadana (“Al estar cerca de áreas rurales, es importante trabajar sobre el riesgo que tienen los productos químicos. Es un área donde no tan lejos fumigan [...]"). Las nociones de "reactivos" y "productos" ("Que al final del curso (sepan) qué reactivos y productos de una reacción química son peligrosos o beneficiosos") y de "proceso" ("Cada reacción representa un proceso y que esos procesos ocurren en la vida cotidiana [...]") son recuperadas por la futura profesora desde esta última perspectiva. Esta reconsideración, de sus concepciones sobre la importancia de enseñar el contenido, es realizada a partir, especialmente, de su experiencia inicial en las aulas, recuperando la relevancia del contexto y expresa la importancia que el Dominio de la Práctica presentó en la construcción de una nueva perspectiva sobre este aspecto de la ReCo.

La ReCo final elaborada por la practicante, a diferencia de la inicial, fue enriquecida con relaciones entre dimensiones asociadas al PCK. ${ }^{\text {ii }}$ Es posible identificar dos relaciones que Revista RBBA Revista Binacional Brasil Argentina 
EL CAMPO DE LA PRÁCTICA DOCENTE EN LA FORMACIÓN INICIAL. UN ESTUDIO

DESCRIPTIVO DE UNA PROPUESTA CURRICULAR PARA LOS PROFESORADOS DE

CIENCIAS EXACTAS Y NATURALES

involucran orientaciones hacia la enseñanza de las ciencias: por un lado, con el conocimiento y creencias acerca de la comprensión de los estudiantes de un tópico específico de la ciencia y el mundo cotidiano ("La capacidad de los alumnos de abordar situaciones de la vida cotidiana con ojo químico"); por otra parte, con las creencias acerca de las estrategias para la enseñanza de la ciencia (“Analogías, modelos y simuladores, para dinamizar el aprendizaje y ayudar al alumno a conceptualizar mejor el tema"). Durante la entrevista, Ana incorpora otras dimensiones como la atención a la diversidad y la responsabilidad docente de crear un contexto en el que se pueda trabajar desde las ideas previas de los estudiantes, entendiendo al aprendizaje como un proceso de redescripción de estas ideas.

Las dificultades consideradas por la practicante para los aprendizajes de los estudiantes en el tema, ganaron en diversidad al transitar de la ReCo inicial a la final. Inicialmente, centró su atención en dificultades asociadas a la dificultad de los estudiantes en "distinguir entre átomos, moléculas y moles" y en "identificar la diferencia entre coeficientes estequiométricos y el número de átomos presentes en la molécula". Estas dificultades, junto a las identificadas como "matemáticas" ("Muchas veces se cometen errores numéricos al balancear y al preguntarles si reconocen el error se ven dificultades para recurrir a las tablas de multiplicar"), son recuperadas por Ana en la ReCo final. En esta última, además, reconoce la relevancia de otras dificultades en los estudiantes: "la predisposición del grupo" y la dificultad “de los alumnos de abordar situaciones de la vida cotidiana con ojo químico". Con relación a esta última, enfatiza en la importancia del "contexto" ("No será significativo utilizar ejemplos que no tengan ningún tipo de relación con la realidad de los educandos") lo que se ve muy de es enfatizado en la entrevista. En particular, la inserción institucional, permitió a la estudiante esta reconsideración del contexto, ejemplificando la relación entre los Dominios de la Práctica y Personal.

Al justificar la relevancia de los conceptos propuestos, es posible reconocer un cambio en términos de considerar la relación con la vida cotidiana, por un lado, y la referencia al empleo de modelos, ambas consideraciones ausentes en la ReCo inicial. Esta nueva lectura del contenido en términos de modelos es justificada por la practicante desde su relevancia epistémica. El trabajo con modelos, referido desde esta última dimensión, no es ejemplificado con el/los modelos a utilizar. La importancia del contenido propuesto se justifica, en ambas instancias, desde su necesidad para la continuidad de la escolaridad. Esta referencia, además, se delimita con mayor claridad en la ReCo inicial a partir de la ejemplificación. En la respuesta 
EL CAMPO DE LA PRÁCTICA DOCENTE EN LA FORMACIÓN INICIAL. UN ESTUDIO

DESCRIPTIVO DE UNA PROPUESTA CURRICULAR PARA LOS PROFESORADOS DE

CIENCIAS EXACTAS Y NATURALES

elaborada para este ítem en la ReCo final la estudiante profundiza su respuesta ejemplificando la relevancia a partir de la consideración explícita de conceptos y procedimientos. En la Tabla 2 se presentan algunas promulgaciones y reflexiones de la estudiante al transitar las Fases.

\begin{tabular}{|c|c|c|c|}
\hline Fase & Tarea & $\begin{array}{l}\text { Promulgaciones } / \text { Ref } \\
\text { lexiones para } \\
\text { relaciones entre } \\
\text { dominios }\end{array}$ & Ejemplos \\
\hline FASE I & $\begin{array}{l}\text { Participación en foros del } \\
\text { aula virtual. (Asignatura: } \\
\text { Teoría de la Educación) }\end{array}$ & $\begin{array}{l}\text { Promulgación del } \\
\text { DPe al DPr }\end{array}$ & $\begin{array}{l}\text { "Desde hace años me parece desastroso } \\
\text { lo que se hace con Salud y } \\
\text { Adolescencia, con } \\
\text { Formación Cívica y con Derecho. Creo } \\
\text { que estas son materias clave para la } \\
\text { población, y están dictadas según planes } \\
\text { muy rígidos que no aportan } \\
\text { herramientas reales a los alumnos..." }\end{array}$ \\
\hline $\begin{array}{l}\text { FASE II } \\
\text { (Inicial) }\end{array}$ & $\begin{array}{l}\text { Respuesta a una pregunta } \\
\text { dentro del instrumento } \\
\text { Reco: ¿Cómo te sentirías } \\
\text { dando el tema? } \\
\text { (Asignatura: Didáctica) }\end{array}$ & $\begin{array}{l}\text { Promulgación del } \\
\text { DPe al DPr }\end{array}$ & $\begin{array}{l}\text { "En cuanto a mí, no puedo negar que mi } \\
\text { educación en el tema se ha visto dada } \\
\text { únicamente en la facultad, considero } \\
\text { que esto me perjudica porque no tengo } \\
\text { ningún recuerdo de cómo veía el tema } \\
\text { en la secundaria y dispongo de un } \\
\text { entendimiento muy tradicional y teórico } \\
\text { con el que debo romper para poder } \\
\text { acercarme a los estudiantes." }\end{array}$ \\
\hline $\begin{array}{l}\text { FASE II } \\
\text { (Inicial) }\end{array}$ & $\begin{array}{l}\text { Respuesta a una pregunta } \\
\text { dentro del instrumento } \\
\text { Reco (¿Qué dificultades } \\
\text { podrían tener los } \\
\text { estudiantes? (Asignatura: } \\
\text { Práctica I) }\end{array}$ & $\begin{array}{c}\text { Reflexión del DC al } \\
\text { DPe }\end{array}$ & $\begin{array}{l}\text { "Cambiar la idea de proceso puede } \\
\text { llegar a ser algo difícil porque uno ya } \\
\text { tiene la idea de la vida cotidiana muy } \\
\text { arraigada". }\end{array}$ \\
\hline $\begin{array}{l}\text { RESIDEN } \\
\text { CIA }\end{array}$ & $\begin{array}{l}\text { Diarios de clase } \\
\text { elaborados durante la } \\
\text { residencia (Asignatura: } \\
\text { práctica de la Enseñanza } \\
\text { II) }\end{array}$ & $\begin{array}{c}\text { Reflexión del DC al } \\
\text { DPr }\end{array}$ & $\begin{array}{l}\text { "Yo quería partir de solución } \\
\text { porque la definición utiliza la } \\
\text { palabra sustancia, pero ellos } \\
\text { sugirieron solución. Decidí probar } \\
\text { de ir por el camino de ellos en vez } \\
\text { de re direccionar hacía el planteo } \\
\text { que yo tenía, como hice en } \\
\text { ocasiones anteriores, pensé que } \\
\text { quizás sería más provechoso } \\
\text { trabajar sobre sus ideas." }\end{array}$ \\
\hline
\end{tabular}

Tabla 2. Ejemplos de promulgaciones y reflexiones realizadas por la futura profesora en cada una de las Fases consideradas.

Revista RBBA Revista Binacional Brasil Argentina 


\section{Reflexiones finales}

En este trabajo presentamos y describimos los cambios proyectados en la estructura curricular para los profesorados de la FCEyN-UNMdP y presentamos, además, un dispositivo para el seguimiento de las trayectorias formativas de los estudiantes. En este contexto, recuperamos resultados parciales de una investigación más amplia centrada en el seguimiento de una futura profesora de Química, que permiten interpretar el trabajo actual en la transición que se desarrolla en diferentes asignaturas y TIPPD en la nueva estructura curricular. Este seguimiento permitió evidenciar cambios en el proceso formativo leídos en términos de interrelaciones entre dominios del MICPD reconocidas en diferentes TIPPD. La adopción del MICPD como dispositivo para el seguimiento de los TIPPD descansa en que recupera dos aspectos que, desde lo formativo, subyacen a la propuesta curricular presentada. Por un lado, entender al crecimiento profesional como un proceso de aprendizaje inevitable y continuo (GUSKEY, 2002). Por otra parte, el MICPD asume la complejidad del crecimiento profesional a través de la identificación de múltiples vías de crecimiento entre los dominios. Su naturaleza no lineal y el hecho de que reconoce el crecimiento profesional como un proceso inevitable y continuo de aprendizaje, distingue este modelo de otros identificados en la literatura de investigación (COENDERS Y VERHOEF, 2019).

La lectura de las trayectorias formativas en términos de cambios entre dominios del MICPD es relevante a efecto de privilegiar una mirada integradora entre las diferentes asignaturas pertenecientes a los trayectos TIPPD y PPD. Los niveles de estructura curricular propuestos por Harden (2000), permitirán, sosteniendo una estructura centrada en disciplinas, avanzar en niveles crecientes de integración considerando la complementariedad en relaciones entre los dominios priorizados en cada asignatura. El MICPD se presentaría, entonces, como un dispositivo - no excluyente pero de significativa importancia- para proporcionar una lectura global de las relaciones entre asignaturas tanto del Campo de la Formación Práctica como de los restantes Campos Formativos. Además, este dispositivo puede guiar decisiones curriculares que permitan fortalecer y ofrecer nuevas interacciones entre dominios que permitan instancias de crecimiento profesional.

Finalmente, el transito del futuro profesional docente por los trayectos correspondientes TIPPD y PPD debería permitirle acceder a variadas instancias para el aprendizaje de la práctica reflexiva en entornos compartidos. Como sostiene DAY (2002), es importante ubicar el Revista RBBA Revista Binacional Brasil Argentina 
EL CAMPO DE LA PRÁCTICA DOCENTE EN LA FORMACIÓN INICIAL. UN ESTUDIO

DESCRIPTIVO DE UNA PROPUESTA CURRICULAR PARA LOS PROFESORADOS DE

CIENCIAS EXACTAS Y NATURALES

aprendizaje profesional a través de la reflexión en el centro del pensamiento crítico y el desarrollo profesional para los docentes. Este desarrollo implica prestar atención a todos los aspectos de la práctica, lo que requiere de un trabajo colectivo, en tanto que la reflexión y la acción conducen a un aprendizaje limitado si es realizado individualmente.

\section{REFERENCIAS}

ALLIAUD, A. La biografía escolar en el desempeño de los docentes. En: SEMINARIO PERMANENTE DE INVESTIGACIÓN DE LA ESCUELA DE EDUCACIÓN DE LA UDESA. 11 de junio de 2007. Buenos Aires. Argentina.

BAENA, M.D. Pensamiento y acción en la enseñanza de las ciencias. Enseñanza de las Ciencias, v. 18, n. 2, p. 217-226, 2000.

CARLSON, J., \& DAEHLER, K. R. The refined consensus model of pedagogical content knowledge in science education. In HUME, A.; COOPER, R.; \& BOROWSKI, A. Repositioning Pedagogical Content Knowledge in Teachers' Knowledge for Teaching. Springer, Singapore, 2019. p. 77-92.

CHENG, M. M. H.; TANG, S. Y.; CHENG, A. Y. N. Practicalising theoretical knowledge in student teachers' professional learning in initial teacher education. Teaching and Teacher EDUCATION, v. 28, n. 6, p. 781-790, 2012.

CLARKE, D.; HOLLINGSWORTH, H. Elaborating a model of teacher professional growth. Teaching and Teacher Education, v. 18, n. 8, p. 947-967, 2002.

COCHRAN-SMITH, M.; LYTLE, S. L. Relationships of Knowledge and Practice: Teacher Learning in Communities. Review of research in education, 24, p. 249-305, 1999.

COENDERS, F.; VERHOEF, N. Lesson study: professional development (PD) for beginning and experienced teachers. Professional development in education, v. 45, n. 2, p. 217-230, 2019.

DAY, C. Developing teachers: The challenges of lifelong learning. Routledge, 2002.

Fogarty, R. Ten ways to integrate curriculum. Educational leadership, v. 49, n. 2, p. 61-65, 1991.

DEWEY, J. Cómo pensamos: nueva exposición de la relación entre pensamiento y proceso educativo. Barcelona: Paidós, 1989. 
EL CAMPO DE LA PRÁCTICA DOCENTE EN LA FORMACIÓN INICIAL. UN ESTUDIO

DESCRIPTIVO DE UNA PROPUESTA CURRICULAR PARA LOS PROFESORADOS DE

CIENCIAS EXACTAS Y NATURALES

GUSKEY, T. R. Professional development and teacher change. Teachers and Teaching, v. 8, n. 3, p. 381-391, 2002.

HARDEN, R. M. The integration ladder: a tool for curriculum planning and evaluation. Medical Education, v. 34, n. 7, p. 551-557, 2000.

HERRERA GONZÁLEZ, J. D.; MARTÍNEZ RUIZ, Á. El saber pedagógico como saber práctico. Pedagogía y saberes (49), p. 9-26, 2018.

HONG, J. Y. Pre-service and beginning teachers' professional identity and its relation to dropping out of the profession. Teaching and Teacher Education, v. 26, n. 8, p. 1530-1543, 2010.

JUSTI, R.; VAN DRIEL, J. The use of the interconnected model of teacher professional growth for understanding the development of science teachers' knowledge on models and modelling. Teaching and Teacher Education, v. 22, n. 4, p. 437-450, 2006.

LOUGHRAN, J., MULHALL, P \& BERRY, A. Understanding and Developing Science Teachers' Pedagogical Content Knowledge. (2da ed.). The Netherlands: Sense Publishers, 2012.

MAINER BAQUÉ, J. La profesionalización docente en perspectiva crítico-genealógica. El caso español. Revista RBBA Vitória da Conquista v. 8, n 2, p. 80-94, 2019.

PERRENOUD, P. (2004). Desarrollar la práctica reflexiva en el oficio de enseñar: profesionalización y razón pedagógica (Vol. 1): Graó.

SANJURJO, L. (2009). Razones que fundamentan nuestra mirada acerca de la formación en las prácticas. In SANJURJO, L. Los dispositivos para la formación en las prácticas profesionales. Homo Sapiens. Rosario. Argentina, 2009, p. 15-43.

SCHÖN, D. (1998). El profesional reflexivo: cómo piensan los profesionales cuando actúan. Barcelona: Paidós

WILSON, C. D.; BOROWSKI, A.; VAN DRIEL, J. (2019). Perspectives on the Future of PCK Research in Science Education and Beyond In HUME, A.; COOPER, R.; \& BOROWSKI, A. Repositioning Pedagogical Content Knowledge in Teachers' Knowledge for Teaching. Springer, Singapore, 2019. p. 289-300.

YAYLI, D. Theory-practice dichotomy in inquiry: Meanings and preservice teacher-mentor teacher tension in Turkish literacy classrooms. Teaching and Teacher Education, 24(4), p. 889-900, 2008. 


\section{Notas}

\footnotetext{
${ }^{i}$ En la asignatura Prácticas Docentes I, los estudiantes elaboran una propuesta de enseñanza y desarrollan simulacros de clases ante sus pares. Ana, realizó estas actividades para la temática indicada, implementando luego la propuesta en un aula. Desarrolló, además, una nueva intervención áulica trabajando con actividades experimentales

ii Entendemos al PCK (conocimiento pedagógico del contenido) según la conceptualización ofrecida en el Refined Consensus Model del PCK (RCM) (véase CARLSON, Y DAEHLER, 2019).
} 